\title{
Bisexual Men Living with HIV: Wellbeing, Connectedness and the Impact of Stigma
}

\author{
Jennifer Power ${ }^{1}$. $\cdot$ Stephanie Amir ${ }^{1} \cdot$ Toby Lea $^{1,2} \cdot$ Graham Brown $^{1,3} \cdot$ Anthony Lyons $^{1} \cdot$ Marina Carman $^{1,4}$. \\ John Rule ${ }^{5}$ Adam Bourne ${ }^{1,6}$
}

Accepted: 13 March 2021 / Published online: 26 March 2021

(c) The Author(s), under exclusive licence to Springer Science+Business Media, LLC, part of Springer Nature 2021

\begin{abstract}
Social research with people living with HIV (PLHIV) rarely distinguishes between gay men and bisexual men. However, bisexual men may have unique experiences of HIV-related stigma and distinct support needs. In this paper, findings are presented from a cross-sectional survey of Australian PLHIV, which included the Berger (HIV) stigma scale. A total of 872 PLHIV completed the survey, of which 48 (6.0\%) were bisexual men. Bisexual men reported higher levels of internalised HIV-related stigma, greater negative self-image and poorer emotional wellbeing than gay men. Bisexual men also reported less social support, less connection with lesbian, gay, bisexual, transgender and queer (LGBTQ) communities, and less connection with other PLHIV. Analysis of data from an open-text question revealed feelings of social isolation and fear of rejection was associated with participant's HIV diagnosis. Study findings suggest that existing social supports for PLHIV may not adequately address the unique support needs of bisexual men.
\end{abstract}

Keywords Bisexuality $\cdot$ Bisexual men $\cdot \mathrm{HIV} \cdot$ Connectedness $\cdot$ Stigma

\section{Introduction}

At the end of 2018 there were approximately 27,000 people living with HIV (PLHIV) in Australia with the number of new diagnoses remaining stable over the past five years at under 1000 per year [1]. Men who have sex with men (MSM) continue to be the main HIV risk exposure group in Australia, accounting for over 60\% of new cases in 2017

Jennifer Power

jennifer.power@latrobe.edu.au

1 Australian Research Centre in Sex, Health and Society, La Trobe University, Building NR6, Bundoora, Melbourne, VIC 3086, Australia

2 Centre for Social Research in Health, UNSW Sydney, John Goodsell Building, Sydney, NSW, Australia

3 Centre for Social Impact, UNSW Sydney, Science Engineering Building, Sydney, NSW, Australia

4 Rainbow Health Victoria, La Trobe University, Building NR6, Bundoora, Melbourne, Australia

5 National Association of People with HIV Australia, Sydney, NSW, Australia

6 Kirby Institute UNSW, UNSW, Wallace Wurth Building, Sydney, Australia
[2]. The national, self-reported HIV prevalence rate among MSM in Australia was 8.2\% in 2019 [3].

Most HIV-related epidemiological research does not differentiate between gay men and bisexual men. In Australia, new HIV cases are recorded in the national surveillance database according to mode of HIV transmission. The transmission category "male to male sex" includes gay and bisexual men if the known route of transmission was sex with another man. However, sexual identity information is not collected which means there is no means to disaggregate data according to sexuality. Even the category "male to male sex" does not adequately capture sexual identity as gay and bisexual men may be listed within the other transmission categories, such as 'injecting drug use' [1]. Social research related to HIV prevention will sometimes differentiate between gay and bisexual men, particularly with respect to whether or not bisexual men engage with safe-sex messaging or whether there are differences between gay and bisexual men in safe-sex behaviours [4]. However, it is more common for gay and bisexual men to be treated as a singular group in behavioural surveillance surveys related to HIV prevention and in social research related to people living with HIV (PLHIV) [5, 6]. There are a range of terminologies used to capture this group: "men who have sex with men", "gay and bisexual men", "gay men, bisexual 
men, and other men who have sex with men" [7, 8]. Even where bisexual men are acknowledged and named as part of the group, the small number of bisexual-identified men relative to the number of gay men means that the experiences of bisexual men are often marginalised or hidden in research or not readily visible in current data collection. While this may not be problematic in population-level studies of HIV transmission, it can be problematic in smaller scale studies which seek to understand human behaviour, safe sex decision making, sexual cultures or the experiences of PLHIV.

There are many reasons why the experiences of bisexual men living with HIV may be different to that of gay men. Historically, bisexual men have held a complex position in relation to HIV. When the epidemic first emerged in the 1980s, bisexual men were often demonised in media and public discourse as they were considered to be the source of HIV transmission to women (who at the time were often positioned as "innocent victims" of HIV) and the heterosexual community in general [7-9]. Alongside this, epidemiological research concerned with the risk of HIV transmission to heterosexual communities [10-12] often implicitly reinforced the notion that bisexual men were problematic due to being a transmission 'link' between gay men and heterosexual women. This complex history means bisexual men may experience a different burden of stigma associated with HIV than gay-identified men. In addition, there is often limited space created for bisexual people within lesbian, gay, bisexual, transgender and queer (LGBTQ) communities [13], which in Australia have been at the forefront of HIV activism and community support [14]. Bisexual men living with HIV are often more isolated from services for PLHIV or less connected to others living with HIV as a result $[15,16]$. Social support acts as a buffer against the negative impact of HIV-related related stigma for PLHIV [17]. Peer support programs, and connection with other PLHIV, may also help to reduce the burden of stigma through creating social interactions where HIV is acknowledged and normalised $[18,19]$ which in turn may reduce negative self-perception associated with HIV [20].

This paper explores the experiences of bisexual men in a sample of Australian PLHIV and compares gay and bisexual men on a range of measures including perceived stigma, social connectedness and mental health. The aim is to better understand the unique needs of the groups most impacted by HIV in Australia in order to target programs to improve health and wellbeing.

\section{Methods}

These data are drawn from the 2015-2016 round of a repeated, cross-sectional survey of people living with HIV (PLHIV) in Australia, the HIV Futures 8 survey, which is conducted every 2.5 years. Full details of the study protocol and method have been published elsewhere [21]. Ethics approval for the study was granted through the La Trobe University College of Science, Health and Engineering Human Ethics Committee (S15-100).

PLHIV, aged 18 years or older and residing in Australia were invited to self-complete a survey in either online or hardcopy form. The survey was advertised widely on social media (namely Facebook), dating apps targeting gay and bisexual men, through HIV organisations, gay community media, HIV-related media, HIV and sexual health clinics, and relevant community events.

\section{Measures}

The survey instrument comprised approximately 250 items containing both closed and open questions. Standard demographic items were used to collect information about age, gender, sexual identity, area of residence, education, employment status and income. HIV-related items included year of diagnosis, current use of antiretroviral treatment, and result of most recent viral load test.

Physical and mental health-related items included: diagnoses of mental health conditions over lifetime, use of medication for a mental health condition in the last six months, general health, emotional wellbeing, resilience and social support. General and mental health were measured using subscales from the RAND 36-Item Short Form Survey (SF36) [22]. Subscale scores range from 0 to 100 and higher scores indicate better health. Resilience was measured with the 10-item Connor-Davidson Resilience Scale [23], with scores ranging from 0 to 40 and higher scores indicating greater resilience. Participants were also asked to describe their overall wellbeing using a single-item categorical measure (excellent, good, fair and poor).

Perceived social support was measured using the 10-item Index of Social Support, in which participants were asked to indicate the extent to which they agreed with statements relating to friendship and support [24, 25]. Summary scores ranged from -30 to 30 and higher scores indicated greater social support. Connectedness to the LGBTQ community was measured using a 6-item adapted version of Frost and Meyer's Connectedness to the LGBT Community Scale [26]. Scores ranged from 6 to 24 and higher scores indicated a stronger sense of community connection. Participants were also asked how much time they spent with other HIV-positive people using a four-point scale (none, a little, some, a lot).

Data on health behaviours relating to smoking, alcohol consumption and non-medical drug use were collected. Alcohol consumption was measured with the Alcohol Use Disorders Identification Test-Consumption (AUDIT-C); scores ranged from 0 to 12 and higher scores indicated a 
higher likelihood of risky alcohol use [27]. Data on nonmedical drug use was collected by asking participants which drugs they had used at least once in the past 12 months. Data on smoking were collected by asking about frequency of tobacco consumption.

Perceived HIV-related stigma was measured using the 40-item Berger HIV Stigma Scale [28]. Scores were calculated for the overall scale (score range 40-160) as well as four established subscales: personalized stigma (relating to the way other have reacted to their HIV status; score range 18-72), concerns about HIV disclosure (score range 10-40), negative self-image (score range 13-52), and concern with public attitudes about people living with HIV (score range 20-80). Higher score indicated greater perceived stigma.

This paper also reports qualitative findings from an opentext question that aimed to elicit people's experiences of HIV-related stigma using specific terms (guilt, shame, fear) that have been associated with stigma in the literature on stigmatised health conditions [29, 30]:

What, if at all, has been your experience of feeling guilty, shameful or fearful about HIV (including fears about how others may react to you being HIV-positive)?

\section{Data Analysis}

Quantitative analyses were conducted using Stata/SE Version 16.1 , and $p<0.05$ was used to determine statistical significance. Chi square tests and $t$ tests were used to compare bisexual men with gay men on sociodemographic characteristics, health and wellbeing, social support and community connection, and perceived HIV-related stigma. Univariable and multivariable linear regression analyses were conducted to determine which variables were associated with perceived HIV stigma (Berger HIV Stigma Scale total score). Variables that were statistically significant in binary comparisons were block entered into the multivariable model.

Out of the 48 bisexual men who participated in the survey 44 (92\%) provided a response to the open text question. Open-text data were analysed using inductive thematic analysis to identify themes relating to the impact of HIV-related stigma on the lives of bisexual men [31]. Data were closely read, initially coded and then grouped into higher level themes for reporting [32]. We only examined responses from bisexual men rather than comparing gay and bisexual men because short text responses provide just a small amount of information and insight into the issues of concern, hence capacity for a nuanced qualitative comparison between two groups is limited. Our analysis of these data is intended to complement the quantitative findings by providing descriptions of how HIV-related stigma is experienced by this sample of bisexual men. Quotations are presented without demographic characteristics in order to protect anonymity.

\section{Results}

\section{Sample}

HIV Futures 8 was completed by 872 participants, which represented approximately $3.5 \%$ of the total number of people living with HIV in Australia in 2015 when survey data were collected [33]. This paper focuses on a subsection of participants, men who identified as bisexual ( $n=48,6.1 \%$ of male participants) or gay $(n=681,87.0 \%$ of male participants). The sample includes cisgender men, transgender men and gender diverse participants who identified as gay. There were eight people who identified as gay and transgender or gender diverse (grouped with gay men for the purposes of this analysis given the focus is on sexual identity), while no bisexual men identified as transgender or gender diverse.

Table 1 shows few differences between the samples of gay men and bisexual men in demographic characteristics. There were no statistically significant differences between gay men and bisexual men in: country of birth $\left(\chi^{2}=0.12\right.$, $\mathrm{p}=0.73)$, Aboriginal and Torres Strait Islander status $\left(\chi^{2}=1.69, p=0.19\right)$, place of living (city/rural) $\left(\chi^{2}=0.01\right.$, $p=0.90)$, highest level of education $\left(\chi^{2}=0.36, p=0.84\right)$, employment status $\left(\chi^{2}=7.44, p=0.19\right)$, reported financial stress $\left(\chi^{2}=3.46, p=0.06\right)$ or relationship status $\left(\chi^{2}=1.20, p=0.75\right)$. Compared to gay men, bisexual men had a higher mean age (55.2 years compared to 49.9, $t=2.81, \mathrm{p}=0.005)$ and were more likely to report their annual income to be AUD $\$ 30,000$ or less $\left(\chi^{2}=14.16\right.$, $p=0.007)$, which at the time of the survey was approximately half the annual average household income in Australia [34]. Of those in a regular relationship, bisexual men were more likely than gay men to report that their primary partner was female (although due to small sample sizes in partner gender categories we cannot report on a statistical comparison) (Table 1).

\section{Quantitative Findings}

\section{Health Status}

There were no differences between gay men and bisexual men with respect to: years living with diagnosed HIV $(t=0.74, \mathrm{p}=0.46)$, use of ART $\left(\chi^{2}=1.15, \mathrm{p}=0.28\right)$, HIV viral load detectability $\left(\chi^{2}=0.86, p=0.36\right)$ or lifetime diagnosis of a mental health condition $\left(\chi^{2}=2.58\right.$, $\mathrm{p}=0.11)$. Compared to gay men, bisexual men reported poorer general health $(t=2.85, \mathrm{p}=0.004)$, poorer emotional wellbeing $(t=2.22, \mathrm{p}=0.03)$ and a higher likelihood of using medication for a mental health condition in the 
Table 1 Sample characteristics by sexual identity

\begin{tabular}{|c|c|c|c|c|}
\hline & Gay men & Bisexual men & Test statistic $\left(\chi^{2}\right.$ or $\left.t\right)$ & $p$ \\
\hline Age (M, SD) & $49.9(12.4)$ & $55.2(12.3)$ & $t=2.81$ & 0.005 \\
\hline Born in Australia (n, \%) & $495(72.7)$ & $36(75.0)$ & 0.12 & 0.73 \\
\hline Aboriginal and/or Torres Strait Islander (n, \%) & $47(6.9)$ & $1(2.1)$ & 1.69 & 0.19 \\
\hline \multicolumn{5}{|l|}{ Residential location (n, \%) } \\
\hline Capital city/suburban & $495(74.2)$ & $36(75.0)$ & \multirow[t]{2}{*}{0.01} & \multirow[t]{2}{*}{0.90} \\
\hline Regional/rural & $172(25.8)$ & $12(25.0)$ & & \\
\hline \multicolumn{5}{|l|}{ Completed education (n, \%) } \\
\hline Up to year 12 & $159(23.5)$ & $12(25.0)$ & \multirow[t]{3}{*}{0.36} & \multirow[t]{3}{*}{0.84} \\
\hline Diploma/trade certificate & $192(28.4)$ & $15(31.3)$ & & \\
\hline University degree & $326(48.2)$ & $21(43.8)$ & & \\
\hline \multicolumn{5}{|l|}{ Current employment (n, \%) } \\
\hline Full-time & $279(41.6)$ & $12(25.0)$ & \multirow[t]{6}{*}{7.44} & \multirow[t]{6}{*}{0.19} \\
\hline Part-time/casual & $104(15.5)$ & $8(16.7)$ & & \\
\hline Student & $29(4.3)$ & $1(2.1)$ & & \\
\hline Retired/pension/home duties & $149(22.2)$ & $15(31.3)$ & & \\
\hline Unemployed & $71(10.6)$ & $7(14.6)$ & & \\
\hline Something else & $39(5.8)$ & $5(10.4)$ & & \\
\hline \multicolumn{5}{|l|}{ Gross annual income (n, \%) } \\
\hline$<\$ 30,000$ & $165(24.2)$ & $18(37.5)$ & \multirow[t]{5}{*}{14.16} & \multirow[t]{5}{*}{0.007} \\
\hline$\$ 30,000-\$ 49,999$ & $97(14.2)$ & $13(27.1)$ & & \\
\hline$\$ 50,000-\$ 99,999$ & $211(31.0)$ & $11(22.9)$ & & \\
\hline$\geq \$ 100,000$ & $162(23.8)$ & $4(8.3)$ & & \\
\hline Prefer not to say / don't know & $46(6.8)$ & $2(4.2)$ & & \\
\hline Financial stress in past $12 \mathrm{~m}(\mathrm{n}, \%)$ & $148(21.7)$ & $16(33.3)$ & 3.46 & 0.06 \\
\hline \multicolumn{5}{|l|}{ Relationship status (n, \%) } \\
\hline Single & $396(59.6)$ & $29(65.9)$ & \multirow[t]{4}{*}{1.20} & \multirow[t]{4}{*}{0.75} \\
\hline Relationship (one person) & $198(29.8)$ & $11(25.0)$ & & \\
\hline Relationship (more than one person) & $62(9.3)$ & $4(9.1)$ & & \\
\hline Something else & $9(1.4)$ & $0(0.0)$ & & \\
\hline \multicolumn{5}{|c|}{ Gender of primary partner (of those in a current relationship; n, \%) } \\
\hline Male & $262(98.1)$ & $8(53.3)$ & \multirow[t]{3}{*}{ N/A due to small cell sizes } & \\
\hline Female & $4(1.5)$ & $7(46.7)$ & & \\
\hline Transgender or gender diverse & $1(0.4)$ & 0 & & \\
\hline
\end{tabular}

previous 6 months $\left(\chi^{2}=7.00, p=0.008\right)$ (Table 2$)$. While there were no statistically significant differences between gay and bisexual men in alcohol consumption $(t=0.08$, $\mathrm{p}=0.94)$ or daily tobacco use $\left(\chi^{2}=0.24, \mathrm{p}=0.89\right)$, bisexual men were less likely than gay men to report non-medical drug use $\left(\chi^{2}=5.64 \mathrm{p}=0.02\right)$ (Table 2).

\section{Social Support and Stigma}

There were no statistically significant differences between gay men and bisexual men in reported levels of resilience $(t=1.45, \mathrm{p}=0.15)$. However, compared to gay men, bisexual men reported lower social support $(t=4.17, \mathrm{p}<0.001)$, less connection to LGBTQ communities $(t=3.91$, $\mathrm{p}<0.001)$ and were less likely to spend time with other
PLHIV $\left(\chi^{2}=10.4, p=0.02\right)$. Bisexual men also reported higher levels of perceived HIV-related stigma across all four stigma subscales-personalised stigma $(t=3.33$, $\mathrm{p}<0.001)$, disclosure $(t=4.33, \mathrm{p}<0.001)$, negative selfimage $(t=4.31, \mathrm{p}<0.001)$ and concern about public attitudes $(t=3.48, \mathrm{p}<0.001)$ as well as the total stigma score $(t=3.86, \mathrm{p}<0.001)($ Table 2$)$.

In the multivariable model, higher total stigma scores were associated with identifying as bisexual $(B=9.25$ $[1.45,17.05], \mathrm{p}=0.02)$, poorer emotional wellbeing $(B=-0.17[-0.28,-0.06], \mathrm{p}=0.003)$, lower social support $(B=-0.64[-0.83,-0.45], \mathrm{p}<0.001)$, lower LGBTQ community connection $(B=-0.81[-1.27,-0.35]$, $\mathrm{p}<0.001)$, and less time spent with PLHIV $(B=-9.26$ $[-15.66,-2.86], \mathrm{p}=0.005)$ (Table 3$)$. 
Table 2 Health and social indicators by sexual identity

\begin{tabular}{|c|c|c|c|c|}
\hline & Gay men & Bisexual men & $\begin{array}{l}\text { Test } \\
\text { statistic }\left(\chi^{2}\right. \\
\text { or } t)\end{array}$ & $p$ \\
\hline Years since HIV diagnosis (M, SD) & $14.6(10.2)$ & $13.5(10.3)$ & 0.74 & 0.46 \\
\hline Currently taking ART (n, \%) & $642(94.3)$ & 47 (97.9) & 1.15 & 0.28 \\
\hline \multicolumn{5}{|l|}{ Last viral load test (n, \%) } \\
\hline Undetectable & $593(87.1)$ & $44(91.7)$ & 0.86 & 0.36 \\
\hline Detectable/unsure & $88(12.9)$ & $4(8.3)$ & & \\
\hline AUDIT-C score (M, SD) & $3.9(2.9)$ & $3.8(3.0)$ & 0.08 & 0.94 \\
\hline \multicolumn{5}{|l|}{ Tobacco use (n, \%) } \\
\hline Smoke daily & $161(24.0)$ & $12(25.5)$ & 0.24 & 0.89 \\
\hline Formerly smoked daily & $252(37.6)$ & $16(34.0)$ & & \\
\hline Never smoked daily & $257(38.4)$ & $19(40.4)$ & & \\
\hline Non-medical drug use in past $12 \mathrm{~m}(\mathrm{n}, \%)$ & $528(77.5)$ & $30(62.5)$ & 5.64 & 0.02 \\
\hline Injecting drug use in past $12 \mathrm{~m}(\mathrm{n}, \%)$ & $94(13.8)$ & $5(10.4)$ & 0.44 & 0.51 \\
\hline Alcohol and other drug treatment in past $12 \mathrm{~m}(\mathrm{n}, \%)$ & $17(2.5)$ & $3(6.3)$ & 2.37 & 0.12 \\
\hline Ever diagnosed with mental health condition (n, \%) & $344(50.5)$ & $30(62.5)$ & 2.58 & 0.11 \\
\hline Prescribed psychiatric medication in past $6 \mathrm{~m}(\mathrm{n}, \%)$ & $202(29.7)$ & $23(47.9)$ & 7.00 & 0.008 \\
\hline \multicolumn{5}{|l|}{ SF-36 (M, SD) } \\
\hline General health & $61.5(24.3)$ & $51.0(27.6)$ & 2.85 & 0.004 \\
\hline Emotional wellbeing & $65.6(21.9)$ & $58.3(24.4)$ & 2.22 & 0.03 \\
\hline Connor-Davidson Resilience Scale (M, SD) & $27.7(8.1)$ & $25.9(9.5)$ & 1.45 & 0.15 \\
\hline \multicolumn{5}{|l|}{ Berger HIV Stigma Scale (M, SD) } \\
\hline Total HIV stigma score & $94.5(24.0)$ & $111.2(21.3)$ & 3.86 & $<0.001$ \\
\hline Personalised stigma & $39.2(12.2)$ & $46.1(11.2)$ & 3.33 & $<0.001$ \\
\hline Disclosure concerns & $27.9(6.9)$ & $32.7(6.1)$ & 4.33 & $<0.001$ \\
\hline Negative self-image & $27.8(8.7)$ & $34.0(8.5)$ & 4.31 & $<0.001$ \\
\hline Concern with public attitudes & $46.8(12.4)$ & $54.2(13.1)$ & 3.48 & $<.001$ \\
\hline Index of Social Support (M, SD) & $9.3(12.4)$ & $0.1(12.5)$ & 4.17 & $<0.001$ \\
\hline LGBTQ community connectedness (M, SD) & $15.8(4.1)$ & $13.2(4.7)$ & 3.91 & $<0.001$ \\
\hline \multicolumn{5}{|l|}{ Time spent with other people living with HIV (n, \%) } \\
\hline None & $187(27.9)$ & $21(44.7)$ & 10.4 & 0.02 \\
\hline A little & $276(41.2)$ & $13(27.7)$ & & \\
\hline Some & $132(19.7)$ & $12(25.5)$ & & \\
\hline A lot & $75(11.2)$ & $1(2.1)$ & & \\
\hline
\end{tabular}

$A R T$ antiretroviral treatment, AUDIT-C Alcohol Use Disorders Identification Test-Consumption, SF-36 36-item short form health survey

\section{Qualitative Findings}

\section{Stigma as Social Isolation Among Bisexual Men}

Bisexual men's responses to the open-text question revealed a profound sense of isolation that was linked to HIV stigma. Participants did not mention bisexuality specifically in relation to their social relationships, rather themes were related to loss of connection with friends and partners as well as withdrawal from the social world.

Several men spoke about retreating from their social lives after their HIV diagnosis due to fear of rejection, as one participant stated, "Since diagnosis, I have very rarely socialised. I very rarely leave my home". Another stated that since their HIV diagnosis and a period of ill-health which required leaving work they had never regained their social networks. They wrote, "I still feel apart from mainstream humanity".

The theme of social isolation extended to romantic and sexual relationships. Many responses related to people feeling that no-one would accept them as a partner and fearing the consequences of disclosing their HIV status, for example the following participant wrote, "I feel that no-one worthy will want me as a partner therefore I will be alone for the rest of my life. I am tired of being asked am I 'clean' by people who want casual sex, this straight away makes me feel like 'damaged goods'. I don't tell people that care for me or love me [about] my status because I feel it will disappoint them and they will look at me differently." 
Table 3 Linear regression analyses examining covariates of HIV stigma (Berger total HIV stigma score)

\begin{tabular}{|c|c|c|c|c|}
\hline & \multicolumn{2}{|l|}{ Univariable } & \multicolumn{2}{|l|}{ Multivariable } \\
\hline & $\mathrm{B}(95 \% \mathrm{CI})$ & $p$ & B $(95 \%$ CI $)$ & $p$ \\
\hline \multicolumn{5}{|l|}{ Sexual identity } \\
\hline Gay & Ref & & Ref & \\
\hline Bisexual & $16.71(8.21,25.22)$ & $<0.001$ & $9.25(1.45,17.05)$ & 0.02 \\
\hline Age & $-0.29(-0.44,-0.14)$ & $<0.001$ & $-0.13(-0.28,0.03)$ & 0.10 \\
\hline Financial stress & $12.90(8.77,17.04)$ & $<0.001$ & $1.15(-3.27,5.57)$ & 0.61 \\
\hline Non-medical drug use in past $12 \mathrm{~m}$ & $-5.86(-10.13,-1.59)$ & 0.007 & $-3.44(-7.72,0.83)$ & 0.11 \\
\hline Prescribed psychiatric medication in past $6 \mathrm{~m}$ & $10.47(6.59,14.35)$ & $<0.001$ & $-0.45(-4.51,3.62)$ & 0.83 \\
\hline \multicolumn{5}{|l|}{ SF-36 subscales } \\
\hline General health & $-0.34(-0.41,-0.27)$ & $<0.001$ & $-0.07(-0.16,0.02)$ & 0.11 \\
\hline Emotional wellbeing & $-0.53(-0.61,-0.46)$ & $<0.001$ & $-0.17(-0.28,-0.06)$ & 0.003 \\
\hline Index of Social Support & $-1.12(-1.25,-0.98)$ & $<0.001$ & $-0.64(-0.83,-0.45)$ & $<0.001$ \\
\hline LGBT community connectedness & $-1.86(-2.31,-1.42)$ & $<0.001$ & $-0.81(-1.27,-0.35)$ & 0.001 \\
\hline \multicolumn{5}{|l|}{ Time spent with other people living with HIV } \\
\hline None & Ref & & Ref & \\
\hline A little & $-15.34(-19.51,-11.16)$ & $<0.001$ & $-7.67(-12.20,-3.15)$ & 0.001 \\
\hline Some & $-20.36(-25.37,-15.35)$ & $<0.001$ & $-7.90(-13.33,-2.48)$ & 0.004 \\
\hline A lot & $-22.48(-28.30,-16.67)$ & $<0.001$ & $-9.26(-15.66,-2.86)$ & 0.005 \\
\hline
\end{tabular}

$S F-36$ 36-item short form health survey

Other participants described a fear of rejection or questioning their own self-worth as a major reason why they avoided seeking sexual or romantic relationships, as one participant said, "I yearn for love but who wants me???" Another participant explained that they similarly have a fear of rejection that they linked to their sense of shame about their HIV diagnosis and fear of unwanted disclosure. They wrote, "I can't start a relationship as the fear of telling someone I love that I have HIV that I would lose them (sic) and that they would tell their family and friends. I feel shameful about having HIV and fearful of people finding out. Don't see much of a future for me-especially no chance of a relationship".

Some participants reported breakdowns in relationships with their spouse or partner after disclosing their HIV status. In some cases, this affected relationships with children or grandchildren. These experiences caused significant distress, and for some remained a source of anguish many years later as one participant wrote, "My wife left me ... She told the two children and their partners without my knowledge and without my being there when they were told... One partner of a son is still having trouble coping with [my HIV status] and this is starting to interfere with [me] seeing my grandson".

\section{Disclosure and Vigilance}

Disclosure, and fear of unwanted disclosure, of HIV status was commonly described by participants as feeling like they held a perpetual sense of vigilance, or social and emotional guardedness, related to fear of rejection. One participant described this, stating: "Everyone talks about their issues, but I feel worried to expose my feelings like normal people. Constant need to be aware of what I'm talking about." Another stated that they "cannot confide in any particular friend as I believe the risk of my disclosure would result in an 'open secret',

Participants explained that they often managed this fear by trying to minimise disclosure, although this required actively avoiding disclosure, as the following participant stated, "I tell no-one. That way they do not have the pressure on them. Once told you cannot un-tell".

Some participants located this fear of disclosure in particular settings, most commonly workplaces: "I have felt fearful at work of having my HIV status disclosed without my consent. I have had this happen to me and then my role at work changed after being 'outed' with weak excuses as to why".

\section{Discussion}

The findings from this study suggest that bisexual men living with HIV in Australia experience a greater sense of social isolation than gay men living with HIV and that this is associated with HIV-related stigma. Bisexual men also felt less connected to LGBTQ communities than gay men and were less likely to have contact with other PLHIV. Given community connectedness and peer support is 
known to be a buffer against the negative impact of stigma on mental health $[35,36]$, these findings suggest that HIVpositive bisexual men may be more vulnerable than gay men to poorer mental health and emotional wellbeing as a result of HIV-related stigma. These findings are significant as they point to a need for social and support programs that are uniquely targeted to bisexual men. Bisexual men may not experience the same benefits from social support programs that broadly target gay and bisexual men as a group.

The finding that bisexual men report poorer emotional wellbeing and greater social isolation than gay men is consistent with previous research on bisexuality in Australia and internationally [37-40]. The reasons for this are not well understood. It is possible that poorer mental health among bisexual people is related to a sense of disconnection from both heterosexual and LGBTQ communities or a response to biphobia and commonly-held negative stereotypes about bisexuality [41, 42]. For bisexual men who are living with HIV, the impact of HIV-related stigma may further exacerbate social disconnection or isolation which in turn may have a negative impact on mental health. A sense of social isolation and lack of support was a core theme in responses to the open text (qualitative) survey questions in this study. These participants linked isolation from friends, families or communities to their HIV status, describing fractures in relationships that occurred as a result of their HIV diagnosis. Experiences of isolation or rejection were especially profound for participants where diagnosis with HIV led to relationship breakdown with partners or children. While it is likely that many gay men may have also experienced a sense of disconnection or rejection from people in their lives as a result of their HIV diagnosis [43], the findings of this study suggest that gay men may be more likely than bisexual men to have access to networks of support and community among other PLHIV or LGBTQ communities. Bisexual men may have less support than gay men to manage the negative impact of HIV-related stigma, which can result in social isolation and loneliness [44].

Vigilance around disclosure of HIV status was a common experience for bisexual men in this study as revealed in open text responses. It is likely that this sense of vigilance and fear about disclosure has a wearing effect on mental health for many PLHIV [45]. Again, without a network of peers, many men in this survey may have limited experience of feeling supported as a person with HIV and limited opportunity to let down their guard in a social setting. Peer support and connection plays a role in reducing the negative impact of HIV-related stigma as it offers PLHIV a sense of solidarity and connection. While peer support interventions may not address stigma at a structural level, they are likely to improve quality of life for PLHIV [20].

\section{Conclusion and Implications for Practice}

Understanding how bisexual men's experience of living with HIV differs from gay men may help provide more tailored support for bisexual PLHIV. It is notable that bisexual men in this survey reported poorer emotional wellbeing, less social connection and higher perceived levels of HIV-related stigma than gay men. This suggests that bisexual men living with HIV may have unique support needs that are not adequately addressed through existing programs [46], and that social and community connection remains a crucial and unmet need for bisexual men living with HIV. Mechanisms to increase social connection should be explored. This most likely includes dedicated initiatives run by and for bisexual PLHIV given their unique experiences with respect to connecting with both the LGBTQ and HIV communities.

More research is needed to understand the drivers of poor mental health outcomes for bisexual people in Australia, and the mechanisms to challenge and address these. Together with existing research into HIV-related stigma, this would allow a more nuanced understanding of the experiences of bisexual men in relation to HIV. Related to this, tackling biphobia and challenging negative stereotypes about bisexuality will reduce the corrosive impact of biphobia on mental health and wellbeing, something that adds an extra burden to the experience of HIV-related stigma and discrimination for bisexual men who are living with HIV. This should be a consideration in policy related to LGBTQ equality and social justice as well as community-level and individuallevel support programs.

Acknowledgements This research would not be possible without the ongoing contribution of people living with HIV. We are grateful to all study participants for their interest and willingness to support this project. The following national HIV organizations and their State and Territory based member organizations contributed to the survey design and recruitment: the National Association of People with HIV Australia, the Australian Federation of AIDS Organisations and the Australasian Society for HIV, Viral Hepatitis and Sexual Health Medicine. Thankyou also The Institute of Many (TIM). Many researchers from the Australian Research Centre in Sex, Health and Society at La Trobe University have worked on this project since 1997. In particular, we acknowledge Michael Bartos, Gary Dowsett, Richard de Visser, Douglas Ezzy, Jeffrey Grierson, Rachel Koelmeyer, Jayne Lucke, Karalyn McDonald, Marian Pitts and Doreen Rosenthal. Thank you to Felicity Rea and Henry von Doussa for assistance with this paper.

Author Contributions JP led the development of the survey instrument and data collection, conceived of the analysis and co-wrote the paper with SA; SA undertook analysis of the open-text data and co-wrote the paper with JP; TL undertook quantitative data analysis and wrote the findings section of the paper; $\mathrm{GB}, \mathrm{JR}, \mathrm{AB}, \mathrm{MC}$ and $\mathrm{AL}$ were actively involved in development of the survey instrument and data collection; all authors reviewed the paper and provided critical commentary on the analysis and text.

Funding This project was funded by the Australian Department of Health. 
Data Availability Data for the HIV Futures study is available on request. Requests can be made to the corresponding author. Appropriate ethics approval will be sought to share data on request.

\section{Declarations}

Conflict of interest The authors declare no conflict of interest.

Ethical Approval Ethics approval for the study was granted through the [removed for blind review].

Informed to Consent All participants completed a survey utilising an online or hardcopy form, completion indicated implied consent as per ethics approval for this study.

\section{References}

1. Kirby Institute. HIV, Viral Hepatitis and Sexually Transmissible Infections in Australia: Annual Surveillance report 2018. Sydney: Kirby Institute, UNSW; 2018.

2. Kirby Institute. HIV, Viral Hepatitis and Sexually Transmissible Infections in Australia Annual Surveillance Report 2017. Sydney Kirby Institute, UNSW; 2017.

3. Mao L, Holt M, Newman C, Treloar C. Annual Report of Trends in Behaviour 2020: HIV and STIs in Australia. Sydney, UNSW Centre for Social Research in Health; 2020

4. Stokes JP, Vanable P, McKirnan DJ. Comparing gay and bisexual men on sexual behavior, condom use, and psychosocial variables related to HIV/AIDS. Arch Sex Behav. 1997;26(4):383-97.

5. Hutchinson JL, Lewis DA, Law M, Bavinton BR, Puhr R, Petoumenos K. Clinical characteristics of overseas-born men who have sex with men (MSM) in the AHOD cohort and implications for clinical practice. Sex Transm Infect. 2020;96(6):469-70. https:// doi.org/10.1136/sextrans-2019-054364.

6. Holt M, Lea T, Mao L, Zablotska I, Lee E, Hull P, et al. Adapting behavioural surveillance to antiretroviral-based HIV prevention: reviewing and anticipating trends in the Australian Gay Community Periodic Surveys. Sex Health. 2017;14(1):72-9.

7. Namaste V, Vukov T, Saghie N, Williamson R, Vallee J, Lafreniére $\mathrm{M}$, et al. HIV prevention and bisexual realities. Canada: University of Toronto Press; 2012.

8. Worth H. The myth of the bisexual infector?: HIV risk and men who have sex with men and women. J Bisex. 2003;3(2):69-88.

9. Power J. Movement, knowledge, emotion: gay activism and HIV/ AIDS in Australia. Canberra: ANU Press; 2011.

10. Ekstrand ML, Coates TJ, Guydish JR, Hauck WW, Collette L, Hulley SB. Are bisexually identified men in San Francisco a common vector for spreading HIV infection to women? Am J Public Health. 1994;84(6):915-9.

11. Zule WA, Bobashev GV, Wechsberg WM, Costenbader EC, Coomes CM. Behaviorally bisexual men and their risk behaviors with men and women. J Urban Health. 2009;86(1):48-62.

12. Chu SY, Peterman TA, Doll LS, Buehler JW, Curran JW. AIDS in bisexual men in the United States: epidemiology and transmission to women. Am J Public Health. 1992;82(2):220-4.

13. Taylor J, Power J, Smith E. Experiences of bisexual identity, attraction, and behavior and their relationship with mental health findings from the who i am study. J Psychosoc Nurs Ment Health Serv. 2020;58(3):28-37.

14. Power J. Movement, knowledge, emotion: gay activism and HIV/ AIDS in Australia. Canberra: ANU Press; 2011. p. 205.
15. Mereish E, Katz-Wise S, Woulfe J. Bisexual-specific minority stressors, psychological distress and suicidality in bisexual individuals: the mediating role of loneliness. Prev Sci. 2017:18:716-25.

16. Herek GM, Capitanio JP, Widaman KF. Stigma, social risk, and health policy: public attitudes toward HIV surveillance policies and the social construction of illness. Health Psychol. 2003;22(5):533.

17. Brener L, Broady T, Cama E, Hopwood M, de Wit JB, Treloar C. The role of social support in moderating the relationship between HIV centrality, internalised stigma and psychological distress for people living with HIV. AIDS Care. 2020;32(7):850-7.

18. Peterson JL, Rintamaki LS, Brashers DE, Goldsmith DJ, Neidig JL. The forms and functions of peer social support for people living with HIV. J Assoc Nurses AIDS Care. 2012;23(4):294-305.

19. Kellett NC, Gnauck K. The intersection of antiretroviral therapy, peer support programmes, and economic empowerment with HIV stigma among HIV-positive women in West Nile Uganda. Afr J AIDS Res. 2016;15(4):341-8.

20. Prati G, Zani B, Pietrantoni L, Scudiero D, Perone P, Cosmaro L, et al. The role of knowing someone living with HIV/AIDS and HIV disclosure in the HIV stigma framework: a Bayesian mediation analysis. Qual Quant. 2016;50(2):637-51.

21. Power J, Brown G, Lyons A, Thorpe R, Dowsett GW, Lucke J. HIV futures 8: protocol for a repeated cross-sectional and longitudinal survey of people living with HIV in Australia. Front Public Health. 2017;5:50. https://doi.org/10.3389/fpubh.2017.00050.

22. Wu AW, Hays RD, Kelly S, Malitz F, Bozzette SA. Applications of the medical outcomes study health-related quality of life measures in HIV/AIDS. Qual Life Res. 1997;6(6):531-54.

23. Connor K, Davidson J. Development of a new resilience scale: the Connor-Davidson resilience scale (CD-RISC). Depress Anxiety. 2003;18(2):76-82.

24. Wilkins RK, Warren D. Families, Incomes and Jobs: A Statistical Report on Waves 1 to 9 of the Household Income and Labour Dynamics in Australia Survey. Melbourne Melbourne Institute of Applied Economic and Social Research, The University of Melbourne; 2012.

25. Baker D. All the lonely people: loneliness in Australia, 20012009. Canberra: The Australia Institute; 2012.

26. Frost DM, Meyer IH. Measuring community connectedness among diverse sexual minority populations. J Sex Res. 2012;49(1):36-49.

27. Dawson DA, Grant BF, Stinson FS, Zhou Y. Effectiveness of the derived alcohol use disorders identification test (AUDIT-C) in screening for alcohol use disorders and risk drinking in the US general population. Alcohol Clin Exp Res. 2005;29(5):844-54.

28. Berger BE, Ferrans CE, Lashley FR. Measuring stigma in people with HIV: psychometric assessment of the HIV stigma scale. Res Nurs Health. 2001;24(6):518-29.

29. Hasson-Ohayon I, Or SE-B, Vahab K, Amiaz R, Weiser M, Roe D. Insight into mental illness and self-stigma: the mediating role of shame proneness. Psychiatry Res. 2012;200(2-3):802-6.

30. Williamson TJ, Ostroff JS, Haque N, Martin CM, Hamann HA, Banerjee SC, et al. Dispositional shame and guilt as predictors of depressive symptoms and anxiety among adults with lung cancer: the mediational role of internalized stigma. Stigma Health. 2020;5(4):425-33.

31. Glaser B. The constant comparative method of qualitative analysis. Soc Probl. 1965;12(4):436-45.

32. Green J, Willis K, Hughes E, Small R, Welch N, Gibbs L, et al. Generating best evidence from qualitative research: the role of data analysis. Aust N Z J Public Health. 2007;31:545-50.

33. Kirby Institute. HIV, Viral Hepatitis and Sexually Transmissible Infections in Australia Annual Surveillance Report 2016. Sydney: Kirby Institute University UNSW; 2016. 
34. Australian Bureau of Statistics. Report number 6302.0: average weekly earnings, Australia, may 2016. Canberra: Australian Bureau of Statistics; 2016.

35. Lyons A, Heywood W. Collective resilience as a protective factor for the mental health and well-being of HIV-positive gay men. Psychol Sex Orientat Gend Divers. 2016;3(4):473-9.

36. Lyons A, Heywood W, Rozbroj T. Psychosocial factors associated with resilience in a national community-based cohort of Australian gay men living with HIV. AIDS Behav. 2016;20(8):1658-66.

37. Bradford $\mathrm{M}$. The bisexual experience: living in a dichotomous culture. In: Fox RC, editor. Current research on bisexuality. New York: Harrington Park Press; 2004. p. 7-23.

38. Bostwick WB, Boyd CJ, Hughes TL, McCabe SE. Dimensions of sexual orientation and the prevalence of mood and anxiety disorders in the United States. Am J Public Health. 2010;100(3):468-75.

39. Leonard W, Lyons A, Bariola E. A closer look at private lives 2. Melbourne: Australian Research Centre in Sex, Health and Society, La Trobe University; 2015.

40. Leonard W, Pitts M, Mitchell A, Lyons A, Smith A, Patel S, et al. Private lives 2: the second national survey of the health and wellbeing of GLBT Australians. Melbourne: Australian Research Centre in Sex, Health and Society, La Trobe University; 2012.

41. Roberts TS. Between a gay and a straight place: bisexual individuals' experiences with monosexism. J Bisex. 2015;15(4):554-69.
42. Dodge B, Herbenick D, Friedman MR, Shick V, Fu T-CJ, Bostwick $\mathrm{W}$, et al. Attitudes toward bisexual men and women among a nationally representative probability sample of adults in the United States. PLoS ONE. 2016;11(10):1-18.

43. Smit PJ, Brady M, Carter M, Fernandes R, Lamore L, Meulbroek $\mathrm{M}$, et al. HIV-related stigma within communities of gay men: a literature review. AIDS Care. 2012;24(4):405-12.

44. Lichtenstein B, Laska MK, Clair JM. Chronic sorrow in the HIVpositive patient: Issues of race, gender, and social support. AIDS Patient Care STDS. 2002;16(1):27-38.

45. Power J, Dowsett GW, Westle A, Tucker JD, Hill S, Sugarman $\mathrm{J}$, et al. The significance and expectations of HIV cure research among people living with HIV in Australia. PLoS ONE. 2020;15(3): e0229733.

46. Pallotta-Chiarolli M, Martin E. "Which sexuality? which service?": bisexual young people's experiences with youth, queer and mental health services in Australia. J LGBT Youth. 2009;6:199-222.

Publisher's Note Springer Nature remains neutral with regard to jurisdictional claims in published maps and institutional affiliations. 